ACTH. Previous trials of ACTH using relatively low dose regimens have proven effective without serious side effects (Millichap JG, et al. JAMA 1962;182:125; Ito M et al. Pediatr Neurol 1990;6:240-244), whereas high-dose, long-term regimens are associated with frequent serious side effects (Snead OC III. Pediatr Neurol 1990;6:147-150).

\title{
LONG-TERM USE OF THE KETOGENIC DIET
}

A retrospective chart review of 28 children (15 males, 14 females) treated with the ketogenic diet for epilepsy for more than 6 years was conducted at Johns Hospital Hospital. Response was documented by attendance at clinic and by telephone. Patients were aged 7 to 23 years at follow-up. Diet duration was 6 to 12 years. Seizure frequency was decreased in $90 \%$ patients. Side effects included kidney stones in $7(25 \%)$, skeletal fractures in $6(21 \%)$, and an increase in the number of children with height below the $10^{\text {th }}$ centile from 10 at initiation of the diet to 23 at follow-up $(p=0.001)$. Overall lipid profiles were generally within the normal range, and cholesterol and triglycerides did not increase significantly. (Groesbeck DK, Bluml RM, Kossof EH. Long-term use of the ketogenic diet in the treatment of epilepsy. Dev Med Child Neurol Dec 2006;48:978-981). (Respond: Eric H Kossof MD, Suite 2158, 200 North Wolfe Street, The Johns Hopkins Hospital, Baltimore, MD 21287).

COMMENT. In this report, the efficacy of the ketogenic diet in the treatment of childhood epilepsy was maintained after long-term use, but side effects, especially growth retardation, kidney stones, and fractures, need to be monitored and may be serious.

Several previous reports of long-term use of the diet, or of adults, 40-50 years of age, previously treated with the ketogenic diet have been published, some from the above authors' institution (Livingston S, 1972) and earlier, from the Mayo Clinic where the diet was first introduced (Keith HM, 1963; Wilder RM, 1921). Livingston allayed concerns about the high fat diet and possible cardiac complications in later life; he found no increased evidence of arteriosclerosis, hypertension, ECG abnormalities, or elevated cholesterol levels in the adults examined. He referred to Haddow Keith, my former colleague and mentor regarding use of the diet, and his report of follow-up data on 530 patients with idiopathic epilepsy treated for periods varying up to 36 years. Of 32 followed for 25 to $30+$ years, 24 were well and 8 improved. Of the total of 530 patients, $30 \%$ were completely free of attacks, $24 \%$ were improved, and $39 \%$ were not benefited; $34(6 \%)$ had died, 4 in status epilepticus.

\section{INFECTIOUS DISEASES}

\section{INFLUENZA A AND FEBRILE SEIZURES}

Predisposing factors and characteristics of febrile seizures in children with influenza A infection were studied in children hospitalized with febrile seizures between January and July 2005 at Tuen Mun Hospital, Hong Kong. Of 177 children confirmed with influenza A infection, $34(19.5 \%)$ had febrile seizures. Ages ranged from 0.9 to 6 years (mean 2.69 years); 19 males and 15 females. Age-matched controls had influenza A but no febrile seizures (control $1 ; n=34$ ), and children with febrile seizures who tested negative for influenza (control $2 ; n=34$ ). Mean maximum body temperature of children with febrile 
seizures and influenza A was $40.4+/-0.67^{\circ} \mathrm{C}$, whereas in those without febrile seizures it was $38.7+/-1.8^{0} \mathrm{C}(\mathrm{P}=0.04)$. Significant factors for development of febrile seizures in influenza included: family history of seizure disorders $(\mathrm{P}=0.03)$; history of febrile seizure $(\mathrm{P}=0.03)$; and coexisting gastroenteritis $(\mathrm{P}=0.05)$. History of febrile seizures was an independent risk factor $(\mathrm{P}=0.015)$. When compared to children with febrile seizures but negative influenza studies, those with confirmed influenza had a significantly higher maximum body temperature, shorter duration of fever before seizure onset, and more frequent occurrence of partial seizures. Current seizure was the first seizure in $26.5 \%$ influenza cases compared to $50 \%$ with negative viral studies $(\mathrm{P}=0.04)$. Febrile seizures were complex in $13(38.2 \%)$ influenza positive vs $7(20.6 \%)$ influenza negative cases. Duration of seizure was longer in the influenza group. (Kwong KL, Lam SY, Que TL, Wong SN. Influenza A and febrile seizures in childhood. Pediatr Neurol Dec 2006;35:395-399). (Respond: Dr Kwong, Department of Paediatrics, Tuen Mun Hospital, Tsing Chung Koon Road, Hong Kong SAR, China).

COMMENT. Influenza A is a frequent cause of febrile seizures in China and Japan but not in the United States and Europe. A recent review of the role of viral infections in the etiology of febrile seizures (Millichap JG, Millichap JJ. Pediatr Neurol Sept 2006;35:165172; idem.J Infect Dis 2004;189:564-565) concluded that fever and the height of the body temperature induced by infection is the essential factor. A threshold convulsive temperature dependent on the height of the body temperature has been established in animal and clinical studies, notably in patients with HHV-6 infection, the most common viral cause in the USA. A higher body temperature is also reported in the above series of influenza-induced febrile seizures. A specific neurotropism and CNS invasive property of HHV-6 is demonstrated in $14.5 \%$ of febrile seizure cases, using CSF-PCR analysis, and a similar neurotropic factor is suggested for influenza A virus. Only $2.5 \%$ HHV-6 cases tested showed CSF pleocytosis, a finding that tends to negate an encephalitic cause. Febrile seizures with influenza A are often complex, and difficult to differentiate from encephalopathy. Systemic immune and cytokine responses to influenza infection have also been invoked as factors. The association of influenza A and febrile seizures is seasonal, related to epidemics, especially prevalent in Asia, sometimes triggered by a concomitant reactivation of HHV infection, and often complicated by encephalopathy or complex seizures. The occurrence of febrile seizures with influenza A epidemics may also be viral strain dependent. Rapid viral diagnostic testing in patients presenting with complex febrile seizures during influenza season is recommended. Effective vaccination may prevent febrile seizures due to influenza.

\section{ENTEROVIRUS 71 BRAINSTEM ENCEPHALITIS AND COGNITIVE AND MOTOR DEFICITS}

Follow-up studies were conducted in 63 previously healthy children with enterovirus 71 brainstem encephalitis ( 49 stage II, 7 stage IIIa, and 7 stage IIIb) at National Cheng Kung University Hospital, Tainan, Taiwan. Mean time to follow-up was $2.8+/-1.0$ years (range 1.4-4.9 years). The ratio of boys to girls was 3 to 2 . Neurologic abnormalities on admission were altered consciousness $(47.6 \%)$, cerebellar dysfunction $(17.5 \%)$, and cranial nerve palsy $(15.9 \%)$. At follow-up, 51 had no deficits, 3 had died, and $9(14.3 \%)$ had residual deficits, cognitive in 3, cerebellar in 7, and severe motor and respiratory failure in 2. (Huang M-C, 